\title{
Impact of COVID-19 on hospitalization of patients with systemic lupus erythematosus (SLE)
}

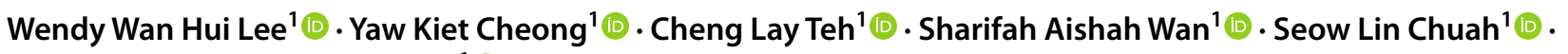 \\ Benjamin Sachdev Manjit Singh ${ }^{1}$ (1)
}

Received: 13 July 2021 / Revised: 6 September 2021 / Accepted: 7 September 2021 / Published online: 11 September 2021

(c) International League of Associations for Rheumatology (ILAR) 2021

\section{Dear Editor,}

Sarawak, the largest Malaysian state on Borneo, recorded its first COVID-19 cases on 13 March 2020, followed by a massive spike in local cases. A strict nationwide lockdown was hence implemented between 18 March to 12 May 2020 to mitigate the spread of COVID-19. Subsequently, the second wave of outbreak came in July 2020, followed by a bigger third wave in late October 2020, with conditional lockdown within the surges. By 31 December 2020, the total number of cases in Sarawak amounted up to 1117 [1]. Sarawak General Hospital, being the largest tertiary hospital (1003 beds) in the state of Sarawak, was designated as a COVID-19 hospital, serving the population of Kuching and its greater territories (population 1,172,618).

The impact of COVID-19 on hospitalization of patients with systemic lupus erythematosus (SLE) has not been well described. We sought to study this issue by comparing SLE hospitalization of Sarawak General Hospital during the pandemic and the previous 5 years (2015-2019). This study was registered in the Malaysian National Medical Research Register (NMRR) and received approval from the Malaysian

Wendy Wan Hui Lee

wendylee1hui@gmail.com

Yaw Kiet Cheong

cykiet@gmail.com

Cheng Lay Teh

tehchenglay@yahoo.com

Sharifah Aishah Wan

shaishah80@yahoo.com

Seow Lin Chuah

celine3732@yahoo.com

Benjamin Sachdev Manjit Singh

benjaminsachdev@gmail.com

1 Rheumatology Unit, Department of Medicine, Sarawak

General Hospital, Sarawak, Malaysia
Medical Research and Ethics Committee (MREC) (NMRR19-2917-51,131 IIR). Clinical characteristics and outcomes of SLE hospitalizations were collected. The primary cause for hospitalization was determined by a physician based on a review of hospitalization records. Standard statistical methods were used to provide descriptive statistics. Cox regression analysis was used to determine the prognostic indicators of mortality in our patients.

A total of SLE admissions were steadily increasing over the years from 2016 to 2019, with a rapid increase from 2018 to 2019 (65.5\%). The total number of SLE patients population in our center up to 31 December 2020 was 620 . Interestingly, there is a significant decline $(45.1 \%)$ in hospitalization in 2020, with an increase in the number of newly diagnosed cases during hospitalizations ( $n=23$ vs. $n=18$ ) as illustrated in Table 1 . The most common reasons for hospitalization remained disease flare $(61.5 \%)$ and infection (32.8\%). There was only one SLE patient admitted for COVID-19 who was discharged well. There was a significant reduction of hospitalization for renal biopsy and renal-related procedures. There was no significant difference in the mean SLEDAI scores among the patients admitted for active SLE in 2020 compared to the previous years. However, there were three cases of severe lupus presenting with gastrointestinal manifestations (one pancreatitis, one superior mesenteric artery thrombosis, one lupus enteritis) and two cases of lupus myocarditis. The number of patients utilizing ICU care was the same as the previous year. Remarkably, the in-hospital mortality rate of SLE patients was higher in $2020(10.7 \%)$ as compared to previous years (3.7-5.4\%). The leading causes of death were flare and infection (6), infection alone (3), and flare alone (3). One patient succumbed to an intracranial bleed. This expanded study reaffirmed our initial findings that the hospitalization rate among SLE patients declined in 2020 but was accompanied by an increase in morbidity and mortality rate [2]. Disease flare and infection remain the 
Table 1 Demographic and clinical characteristics of SLE patients and hospitalization pattern in Sarawak General Hospital from year 2015 to 2020

\begin{tabular}{|c|c|c|c|c|c|c|}
\hline & 2015 & 2016 & 2017 & 2018 & 2019 & 2020 \\
\hline Total admissions & 106 & 105 & 118 & 134 & 222 & 122 \\
\hline Change in total admissions (\%) & NA & -1 & +12 & +13.5 & +65.5 & -45.1 \\
\hline Total patients & 78 & 76 & 84 & 98 & 127 & 85 \\
\hline Total readmission & 24 & 28 & 28 & 25 & 39 & 26 \\
\hline Gender (female:male) & $74: 4$ & $70: 6$ & $73: 11$ & $86: 12$ & $114: 13$ & $76: 9$ \\
\hline Age at admission in years, mean (SD) & $32.41(13.88)$ & $32.66(12.47)$ & $33.64(12.86)$ & $34.26(14.21)$ & $34.72(15.09)$ & $38.28(14.43)$ \\
\hline Duration of SLE in months, mean (SD) & $83.97(86.48)$ & $50.78(66.76)$ & $71.93(77.11)$ & $83.71(95.51)$ & $86.87(91.78)$ & $82.26(99.43)$ \\
\hline SLICC damage score, mean (SD) & $0.48(1.05)$ & $0.22(0.60)$ & $0.51(1.11)$ & $0.60(1.26)$ & $0.87(1.56)$ & $0.62(1.24)$ \\
\hline Nephritis, $n(\%)$ & $47(60.3)$ & $54(71.1)$ & $54(64.3)$ & $70(71.4)$ & $102(80.3)$ & $63(74.1)$ \\
\hline Antiphospholipid syndrome, $n(\%)$ & $5(6.4)$ & $9(11.8)$ & $9(10.7)$ & $10(10.2)$ & $17(13.4)$ & $7(8.2)$ \\
\hline Hypertension, $n(\%)$ & $25(32.1)$ & $24(31.6)$ & $16(19.0)$ & $34(34.7)$ & $40(31.5)$ & $33(38.8)$ \\
\hline End stage renal failure, $n(\%)$ & $15(19.2)$ & $13(17.1)$ & $18(21.4)$ & $29(29.6)$ & $41(32.3)$ & $13(15.3)$ \\
\hline \multicolumn{7}{|l|}{ Medications, $n(\%)$} \\
\hline Hydroxychloroquine & $66(84.6)$ & $58(76.3)$ & $78(92.9)$ & $81(82.7)$ & $119(93.7)$ & $58(68.2)$ \\
\hline Steroid & $60(76.9)$ & $54(71.1)$ & $64(76.2)$ & $70(71.4)$ & $113(89.0)$ & $54(63.5)$ \\
\hline Immunosuppressant & $46(59.0)$ & $37(48.7)$ & $49(58.3)$ & $50(51.0)$ & $83(65.4)$ & $50(58.8)$ \\
\hline Total new cases diagnosed during hospitalizations & 17 & 21 & 8 & 17 & 18 & 23 \\
\hline Total deaths & 5 & 4 & 2 & 6 & 8 & 13 \\
\hline In-hospital mortality rate $(\%)$ & 4.7 & 3.8 & 5.0 & 3.7 & 5.4 & 10.7 \\
\hline Length of stay in days, mean (SD) & $8.43(8.33)$ & $7.85(9.51)$ & $7.42(8.79)$ & $6.70(6.17)$ & $6.97(9.02)$ & $8.17(8.55)$ \\
\hline Total patient with ICU stays & 7 & 2 & 6 & 5 & 12 & 12 \\
\hline \multicolumn{7}{|l|}{ Reason for admission, $n(\%)$} \\
\hline 1. Flare & $51(48.1)$ & $60(57.1)$ & $47(39.8)$ & $56(41.8)$ & $71(32.0)$ & $55(45.1)$ \\
\hline 2. Flare and infection & $22(20.8)$ & $18(17.1)$ & $13(11.0)$ & $20(14.9)$ & $15(6.8)$ & $20(16.4)$ \\
\hline 3. Infection & $24(22.6)$ & $20(19.0)$ & $18(15.3)$ & $25(18.7)$ & $40(18.0)$ & $20(16.4)$ \\
\hline 4. Others & $9(8.5)$ & $7(6.7)$ & $40(33.9)$ & $33(24.6)$ & $96(43.2)$ & $27(22.1)$ \\
\hline Total admission for flare, $n(\%)$ & $73(68.9)$ & $78(74.3)$ & $60(50.8)$ & $76(56.7)$ & $86(38.7)$ & $75(61.5)$ \\
\hline SLEDAI score, mean (SD) & $8.97(5.03)$ & $7.97(4.33)$ & $7.98(6.01)$ & $8.21(5.34)$ & $8.53(4.72)$ & $8.84(5.42)$ \\
\hline Total admission for infection, $n(\%)$ & $46(43.4)$ & $38(36.2)$ & $31(26.3)$ & $45(33.6)$ & $55(24.8)$ & $40(32.8)$ \\
\hline Renal biopsy, $n(\%)$ & $17(16.0)$ & $11(10.5)$ & $21(17.8)$ & $21(15.7)$ & $32(14.4)$ & $13(10.7)$ \\
\hline Renal replacement procedures, $n(\%)$ & $0(0.0)$ & $3(2.9)$ & $2(1.7)$ & $0(0.0)$ & $34(15.3)$ & $4(3.3)$ \\
\hline
\end{tabular}

leading causes of admission and mortality among our SLE patients. Analysis using Cox regression showed that there were only two independent predictors of mortality, namely the presence of infection (HR 6.11) and age (HR 1.03).

The increasing trend of SLE hospitalization over the years from 2016 to 2019 could be attributed to the expanding rheumatology service and the rising number of SLE referrals with the improvement of knowledge and awareness of SLE among general practitioners and physicians. This was however followed by a significant drop of SLE hospitalization in 2020 - the year of COVID-19 spread with its resulting impact on various aspects of life. There was only one SLE patient who was admitted with COVID-19, and she was discharged well. This concurred with the study by Espinosa $\mathrm{G}$ et al. [3] which showed that the incidence of COVID-19 is low in a cohort of SLE patients with stable and clinical inactive disease and all SLE patients with confirmed SARSCoV-2 infection in their study recovered.

Measures instituted to mitigate the pandemic, such as lockdowns, rescheduling of non-urgent outpatient appointments, and the restructuring of medical care towards COVID-19, had limited patients' access to healthcare and disrupted routine medical care. These measures resulted in diagnostic delay and suboptimal medical care as forewarned by Horisberger et al. [4]. Routine care cancelations and difficulties in accessing healthcare also contributed to physical deterioration among patients with lupus [5]. Additionally, avoidance of care by patients due to fear of the pandemic had led to a higher risk of disease flare and severe illness at presentation. Furthermore, social determinants of health such as food security, income, and social isolation impose adverse psychosocial effects on patients during this pandemic $[6,7]$. 
Although telemedicine has been widely used to replace physical clinic visits during this pandemic, it might be insufficient especially for patients with SLE, as close monitoring of both clinical and laboratory parameters is essential in the detection of flares and deterioration. Fernandez-Ruiz R et al. [8] highlighted that patients with SLE require routine monitoring of laboratory parameters and that new patients or those with urgent concerns may require a more thorough evaluation than what is feasible by telemedicine.

Our study showed that the rate of SLE hospitalization declined during this pandemic, but it was accompanied by an increased mortality rate due to measures implemented to mitigate the pandemic. As our hospital was a designated COVID-19 hospital, our data has its inherent selection bias. Further studies are needed to ascertain the impact of COVID-19 on the pattern of hospitalization among SLE patients worldwide. Nevertheless, special attention must be given to our vulnerable population of patients when implementing new measures to curb the pandemic. All this is in the hope of delivering more effective healthcare for our patients during this current pandemic and in preparation for future health crises.

Acknowledgements The authors would like to thank the Director General of Health, Malaysia, for permission to publish this article.

Author contribution Lee WWH analyzed data, did the literature search and wrote the manuscript. Cheong YK acquired the data and wrote the manuscript. Teh CL conceived the study, analyzed data, and critically revised the manuscript. Wan SA, Chuah SL, and Sachdev Manjit Singh $\mathrm{B}$ acquired the data and critically revised the manuscript. All authors approved the final version of the paper.

Data availability All data generated or analyzed during this study are included in this published article.

\section{Declarations}

Disclosures None.

\section{References}

1. Sarawak state disaster management committee initiative website. Available: https://sarawak.gov.my/web/home/article

2. Chuah SL, Teh CL, Wan Mohd Akbar SA et al (2020) Impact of COVID-19 pandemic on hospitalisation of patients with systemic lupus erythematosus (SLE): report from a tertiary hospital during the peak of the pandemic. Ann Rheum Dis annrheumdis-2020-218475. Advance online publication. https://doi.org/10. 1136/annrheumdis-2020-218475

3. Espinosa G, Prieto-González S, Llevadot M et al (2021) The impact of SARS- CoV-2 coronavirus infection in patients with systemic lupus erythematosus from a single center in Catalonia. Clin Rheumatol 40:2057-2063. https://doi.org/10.1007/ s10067-021-05675-x

4. Horisberger A, Moi L, Ribi C et al (2020) Impact of COVID-19 pandemic on SLE: beyond the risk of infection. Lupus Science \& Medicine 7:e000408. https://doi.org/10.1136/lupus-2020-000408

5. Sloan M, Gordon C, Harwood R et al (2020) The impact of the COVID-19 pandemic on the medical care and health-care behaviour of patients with lupus and other systemic autoimmune diseases: a mixed methods longitudinal study. Rheumatology Advances in Practice 5(1): rkaa072. https://doi.org/10.1093/rap/ rkaa072

6. Rathi M, Singh P, Bi HP et al (2021) Impact of the COVID-19 pandemic on patients with systemic lupus erythematosus: observations from an Indian inception cohort. Lupus 30(1):158-164. https://doi.org/10.1177/0961203320962855

7. Mehta B, Jannat-Khah D, Fontana MA et al (2020) Impact of COVID-19 on vulnerable patients with rheumatic disease: results of a worldwide survey. RMD Open 6(3):e001378. https://doi.org/ 10.1136/rmdopen-2020-001378

8. Fernandez-Ruiz R, Paredes JL, Niewold TB (2021) COVID-19 in patients with systemic lupus erythematosus: lessons learned from the inflammatory disease. Translational research: the journal of laboratory and clinical medicine 232:13-36. https://doi.org/10. 1016/j.trs1.2020.12.007

Publisher's note Springer Nature remains neutral with regard to jurisdictional claims in published maps and institutional affiliations. 